\title{
Burnout Toll on Empathy Would Mediate the Missing Professional Support in the COVID-19 Outbreak
}

\author{
Cristina Bredicean, ${ }^{1,2}$ \\ Simona Claudia Tamasan, ${ }^{3}$ \\ Diana Lungeanu, (D) \\ Catalina Giurgi-Oncu, (iD ${ }^{1,5}$ \\ lleana-Pepita Stoica, ${ }^{6}$ Anca- \\ Livia Panfil, ${ }^{3}$ Claudia Vasilian, ${ }^{3}$ \\ Ica Secosan, 7 Sorin Ursoniu, ID 5,8 \\ Raul Patrascu, \\ 'NEUROPSY-COG Center for Cognitive \\ Research in Neuropsychiatric Pathology, \\ Department of Neuroscience, "Victor Babes" \\ University of Medicine and Pharmacy, \\ Timisoara, Romania; ${ }^{2}$ Psychiatry \\ Compartment, "Dr. Victor Popescu" \\ Emergency Military Clinical Hospital, Timisoara, \\ Romania; 'Liaison Psychiatry, "Pius Branzeu" \\ County Emergency Hospital, Timisoara, \\ Romania; ${ }^{4}$ Center for Modeling Biological \\ Systems and Data Analysis, Department of \\ Functional Sciences, "Victor Babes" University \\ of Medicine and Pharmacy, Timisoara, Romania; \\ 5"Pius Branzeu" County Emergency Hospital, \\ Timisoara, Romania; ${ }^{6}$ Mental Health Center, \\ "Pius Branzeu" County Emergency Hospital, \\ Timisoara, Romania; ${ }^{7}$ Department of Plastic \\ and Reconstructive Surgery, "Pius Branzeu" \\ County Emergency Hospital, Timisoara, \\ Romania; ${ }^{8}$ Discipline of Public Health and \\ Health Management, Center for Translational \\ Research and Systems Medicine, Department of \\ Functional Sciences, "Victor Babes" University \\ of Medicine and Pharmacy, Timisoara, Romania; \\ ${ }^{9}$ Department of Functional Sciences, "Victor \\ Babes" University of Medicine and Pharmacy, \\ Timisoara, Romania
}

Correspondence: Diana Lungeanu Center for Modeling Biological Systems and Data Analysis, Department of

Functional Sciences, "Victor Babes"

University of Medicine and Pharmacy,

Piata Eftimie Murgu 2, Timisoara, 30004I, Romania

Tel +40-256-I 90288

Fax +40-256-190288

Email dlungeanu@umft.ro

Catalina Giurgi-Oncu

NEUROPSY-COG Center for Cognitive

Research in Neuropsychiatric Pathology,

Department of Neuroscience, "Victor

Babes" University of Medicine and

Pharmacy, Piata Eftimie Murgu 2,

Timisoara, 30004I, Romania

Email catalina.giurgi@umft.ro
Purpose: The year 2020 was marked by the COVID-19 pandemic, massively disruptive at the general population level and for healthcare systems. We aimed to evaluate the psychological distress associated with work-related experiences among medical professionals and supporting staff during the pandemic outbreak.

Patients and Methods: A cross-sectional survey was conducted between April and May 2020, employing a self-administered on-line questionnaire that included the collection of socio-demographic and professional status information, Hamilton Anxiety Rating Scale, nine-item Patient Health Questionnaire (PHQ-9), Maslach Burnout Inventory - General Survey, Connor-Davidson Resilience Scale, and a subscale of the International Personality Item Pool (IPIP) for empathy. A total of 364 professionals of the county-coordinated area responded. Descriptive statistics summarized the findings and a mediation model was analyzed, applying the causal step strategy. The specific direct and causal mediation effects were estimated with the bias-corrected and accelerated bootstrap sampling method.

Results: Anxiety, burnout, stress, resilience, and empathy proved to be significantly associated with both the professional category (ie, consultant, specialty doctor, trainee doctor, senior nurse, trainee nurse or other) and the perceived professional support (the nonparametric multivariate permutation test resulted in $\mathrm{p}=0.048$ and $\mathrm{p}<0.001$, respectively). When controlling for the marital and professional status, the female gender had an $\mathrm{OR}=2.26,95 \%$ CI $(1.21 ; 4.22)$ toward a high level of empathy compared to males. The causal mediation effect of the perceived lack of professional support on empathy through burnoutdepersonalization was highly significant $(\mathrm{p}<0.001)$ with an average of $0.0599,95 \%$ CI $(0.0238 ; 0.10)$, while the direct effect was non-significant $(\mathrm{p}=0.536)$ with an average of $0.0295,95 \%$ CI $(-0.0774 ; 0.15)$.

Conclusion: We highlighted the impact of the lack of professional support on healthcare workers' empathy through burnout-depersonalization in a context of a high workload, time pressure, job stress beyond previous training, and inherently limited organizational support, which are potentially modifiable factors in the mid-term.

Keywords: healthcare workers, burnout experience, work engagement, mediation model, Maslach Burnout Inventory, PHQ-9

\section{Introduction}

On 11 March 2020, the World Health Organization characterized COVID-19 outbreak as a pandemic. ${ }^{1}$ When faced with such a major crisis, our mental health can be affected. On the other hand, some mental health-related characteristics help us resist and survive. Constant worries regarding uncontrollable threats may lead to anxiety, depression, and unhealthy responses to stress, reducing tolerance and empathy. ${ }^{2-5}$ Exacerbated by the accompanying infodemic, ${ }^{6}$ the negative 
consequences on medical staff are expected to increase in such difficult working conditions.

Stress is defined as the assessment of a situation that exceeds coping resources, ${ }^{7}$ and is an issue that frequently occurs in the healthcare professionals. ${ }^{7,8}$ Research shows that stress might cause symptoms of anxiety, depression, ${ }^{9,10}$ emotional exhaustion, ${ }^{11}$ tense relationships, ${ }^{12}$ and lower job satisfaction. ${ }^{8}$ These issues correlate with a higher frequency of burnout, ${ }^{13}$ and a lower quality of life. ${ }^{14}$ During previous disease outbreaks, the healthcare workers manifested psychological distress and psychiatric symptoms, such as anxiety, depression and stress, fear of self-infection, concern about infecting family and friends, uncertainty and stigmatization, all with possible long-term psychological implications. ${ }^{15-17}$

Empathy is a fundamental value for the healthcare, enabling professionals to understand and share a patient's feelings and perspective. ${ }^{16}$ Patients prefer to relate to professionals who are competent, but also have adequate interpersonal and emotional skills. ${ }^{9,15-22}$ Research has shown that patients' trust in medical staff, and empathic care lead to greater adherence to treatment and better health outcomes. ${ }^{23,24}$ Doubtless, empathy is a core element of effective care, although it also creates vulnerability to stress, emotional exhaustion, and burnout. ${ }^{25-27}$ Most authors see the concept as having two dimensions (ie, emotive, cognitive), but Wilkinson et $\mathrm{al}^{18}$ described it as having two additional dimensions (ie, behavioral and moral) that should be targeted for organizational cultural interventions.

Burnout is defined as physical exhaustion, helplessness, decreased motivation to work or live, usually as a result of prolonged stress or frustration. ${ }^{28-31}$ Burnout among healthcare professionals is a gradual process developing over time and has been characterized as a major concern. ${ }^{32-37}$ The relationship between burnout and empathy is widely acknowledged, but there have been inconclusive research results in establishing the direction and nature of this relationship, ${ }^{38-40}$ with widely accepted evidence supporting a negative correlation. ${ }^{18,41,42}$ Burnout is related to the stress of one's professional activity, while empathy is an individual human trait; three current hypotheses are explored in related research: exhaustion decreases the ability of medical staff to respond empathetically, being empathetic can lead to exhaustion, and being empathetic protects against burnout.

Perceived organizational support evaluates employees' perception and acknowledgement of effective role fulfillment, ${ }^{43}$ and was identified as a moderator for the relationship between work and work-related outcomes. ${ }^{44}$ Successful planning and implementation of interventions towards supporting healthcare workers' mental health, reducing physicians' vulnerability, and preparing for health emergencies has been a constant concern. ${ }^{34,45-47}$

The outbreak of the COVID-19 pandemic was a period of high pressure on healthcare workers and heavy strain on the healthcare system's resources. The goals of this research were to: (1) assess the psychological distress associated with work-related experiences during the outbreak; (2) evaluate the burnout during the outbreak; (3) identify the modifiable factors associated with empathy among healthcare workers during that period.

\section{Patients and Methods Study Design and Participants}

An online cross-sectional survey was employed for data collection. It was distributed to healthcare professionals working under the supervision of the county-level healthcare authority and the professional coordination of the "Pius Branzeu" Emergency Hospital, a tertiary hospital affiliated with "Victor Babes" University of Medicine and Pharmacy in Timisoara, Timis County, Romania. The study was approved by the Ethics Committee of the "Pius Branzeu" Emergency Hospital (ID 191, assigned on 5 Mar 2020) and it complies with the Declaration of Helsinki. Data collection and analysis followed the European Union's General Data Protection Regulation (GDPR). The participants were recruited via their professional contact information, and the survey started with information about the study's goals and personal data protection. For each individual, data collection proceeded after informed consent had been granted by participants (a required confirmation was included as the first questionnaire's item). First-contact professionals were asked to disseminate the information and the survey link. The online data collection was active for 20 days, between 28 April and 17 May 2020, and ended when there were two consecutive days with only one respondent. In total, the survey gathered information from 364 professionals (ie, doctors, nurses, and administrative staff) with a response rate of $12.62 \%$ (out of 2884 total targeted staff). Administrative and clerical personnel were included for their important role in coordinating and facilitating patient care. The data form did not require any identification information (ie, anonymous) but 
offered the option to fill-in an e-mail address for receiving personalized feedback and the possibility of further contact. All the other items were required. Less than $40 \%$ of the respondents provided identification information, with no distinct patterns across gender, age, or professional status.

The online form was created on a survey platform and gathered socio-demographic data (ie, age group, gender, marital status); information on the level of career, professional responsibilities, and whether the respondents were on the frontline of pandemic engagement; indicators of stress and psychological functioning; perceived support in the professional and family environment (both as dichotomic variables).

\section{Psychological Assessment Instruments}

The 14-item Hamilton Anxiety Rating Scale (HARS) was used to assess the severity of perceived anxiety symptoms. ${ }^{48}$ On HARS, answers are rated on a 5-point scale, summing a total score that ranges from 0 to 70 . HARS is a clinician-based questionnaire that may also be employed as a self-scored survey.

Depression was measured with the 9-item Patient Health Questionnaire (PHQ-9), validated for the Romanian population. ${ }^{49,50}$ On PHQ-9, each of the nine items is scored by the subject on a scale from 0 ("not at all") to 3 ("nearly every day"). The total possible score ranges from 0 to 27 points, with values over 14 as a trend toward severe depression.

Experienced burnout was assessed with the 16-item Maslach Burnout Inventory - General Survey (MBI-GS), including three dimensions: emotional exhaustion (five items), cynicism-depersonalization (five items), and diminished professional efficacy (six items). ${ }^{31,51}$ The MBI-GS was previously used for the assessment of Romanian healthcare professionals. ${ }^{51}$

The 25-item Connor-Davidson Resilience Scale (CDRISC) was used to gauge the respondents' levels of resilience. ${ }^{52}$ It had been translated and used with the Romanian population. ${ }^{53}$

A subscale of the International Personality Item Pool (IPIP) was employed for empathy. ${ }^{54}$ The Romanianvalidated version IPIP-Ro was applied, on a scale from 1 to 8 (the higher the score, the higher the level of empathy). ${ }^{55}$ The actual measurements' distribution was strongly skewed towards the high end of the scale, with almost $80 \%$ of the values at the maximum eight, thus having little discriminating power over the scale range.
Therefore, we decided to further analyze this assessment as a binary variable: having/showing either empathy (the maximum score on the scale) or little/lack of empathy (smaller scores).

\section{Data Analysis}

Descriptive statistics included the observed frequency counts (percentage) for category variables and median (Inter Quartile Range) for numerical variables. Univariate non-parametric statistical tests were applied to compare the distribution of the questionnaires' scale data across two or multiple groups, as appropriate (either MannWhitney U or Kruskal-Wallis tests, respectively). The Chi-square statistical test (either asymptotic or MonteCarlo simulation with 10,000 samples) was applied to check the statistical significance of the association between the category variables. A multivariate nonparametric model was employed for investigating the effect of working conditions and professional or socio-demographic factors on the questionnaires' measurements, when the outcome variables proved statistically significant in preliminary univariate analysis of those factors. The ShapiroWilk statistical test (in both uni- and multivariate versions) was employed for testing the normality, and the Box's test was used for checking the multivariate homogeneity. For the nonparametric multivariate testing (when the assumptions for the MANOVA test were not met), a permutation simulation with 1000 replicates was applied to obtain the reported p-values.

A stepwise logistic regression model was applied with empathy as the outcome, and the working conditions, professional and socio-demographic factors, or questionnaires' scale data as possible independent predictors, based on the preliminary univariate analysis (when $\mathrm{p}<0.15){ }^{56}$ The Akaike information criterion (AIC) was used to select the best fitting model when conducting the logistic regression.

A mediation model was analyzed, with empathy as the outcome and the causal step strategy was employed for testing the mediation hypotheses. ${ }^{57-59}$ For estimating the specific direct and causal mediation effects, the biascorrected and accelerated bootstrap re-sampling strategy (with 1000 bootstrap samples) was applied. ${ }^{59,60}$

For all scales, the actual reliability of the measurements was assessed based on Cronbach's alpha. Values > 0.8 were considered as proving good internal consistency for this applied research. ${ }^{61}$ 
The statistical analysis was conducted at a $95 \%$ level of confidence and a 5\% level of statistical significance. All reported probability values were two-tailed. Statistical analysis was performed with the statistical software IBMSPSS v. 25 and R 3.6.3 packages (including "mvnormtest", "ICSNP", “npmv", and "mediation").

\section{Results}

Three hundred and sixty-four professionals were included in this study ( 73 males, $20.1 \%$ ) and approximately $60 \%$ of them were between 31 and 50 years old. Table 1 shows the descriptive statistics for the socio-demographic and professional data. We considered the current professional status as comprising information on the age group and level of education, therefore the subsequent statistical analysis was conducted using this comprehensive variable. Table 2 presents the descriptive statistics for the scale measurements: the resilience and the empathy proved to be different for the two genders.

Of the 364 respondents, $14.83 \%$ were slightly depressed (PHQ-9 scores over 4) and $29.67 \%$ reported an anxious mood (HARS scores over 4), with higher scores on both scales among those who operated in the frontline "red area", although no evident relationship was found between levels of professional responsibility (coordinators versus non-coordinators). Tables 3 and 4 show the scales' scores in relation to professional status (with a statistically significant association for most of them), the coordination responsibilities (higher resilience for coordinators, with highly significant difference, $\mathrm{p}=0.004$ ), and the activity in frontline "red area" (higher PHQ-9 scores, statistically significant for the frontline workers, $\mathrm{p}=0.018$ ). Table 5 presents the association of perceived support with the psychological scales' scores: the lack of support from the professional environment seemed decisive for higher anxiety and stress, and less resilience and empathy, with highly significant statistical association $(\mathrm{p}<0.01)$. The multivariate testing proved the statistically significant association of the scales' measurements with the professional categories, activity in COVID19 red areas, and professional support.

The scales' measurements showed no relationship with the respondents' desire to receive personalized feedback (thus providing their identity and contact information), except for the resilience scale: those requesting feedback proved to be more resilient (Mann-Whitney $U$-test, $\mathrm{p}=0.007$ ).

Table 6 shows the results of the step-wise logistic regression analysis with empathy as the outcome. Models $0,1,2$, and 3 were built applying a step-wise logistic regression employing AIC for model selection. Based on Model 3, the odds of empathy among women were more than twice those among men $(2.26=1 / 0.442)$ for healthcare professionals with the same burnout-depersonalization scoring, perceived professional support, total scoring on the resilience scale, professional status, and marital status: $\mathrm{OR}=2.26$, $95 \%$ CI $(1.21 ; 4.22)$. Each additional scoring point on the burnout-depersonalization scale decreased the empathy odds at a ratio of $\mathrm{OR}=0.93,95 \% \mathrm{CI}(0.89 ; 0.97)$ while controlling for other factors in the model.

The lack of professional support accounted for higher Maslach B depersonalization scoring, with high statistical significance (Table 5): median (Inter Quartile Range) of 2 $(0-6)$ versus $7(2-14)$ for the groups who acknowledged as receiving professional support versus those who did not, respectively. Figure 1 is a graphical representation of the triad-type relationship between professional support, burnout-depersonalization, and empathy or lack of empathy.

Taking all these into consideration, the causal mediation model of burnout-depersonalization role on the association between professional support and the empathy of healthcare professionals is proposed (shown in Figure 2). For estimating the specific direct and causal mediation effects of professional support on empathy, for which this model accounts, the bias corrected and accelerated (BCa) bootstrap resampling was applied to determine the confidence intervals. Table 7 and Figure 3 present the results. The total effect of the professional support on empathy was mainly generated through the mediation mechanism, ie burnout-depersonalization in the case of no professional support. The causal mediation effect was highly significant, while the direct effect was negligible. The interaction between the perceived professional support and burnout was non-significant (as it was proven in the step-wise logistic regression analysis, as well).

\section{Discussion}

In Romania, there are certain work-related conditions that have changed. Since the beginning of the pandemic, all Romanian hospitals were organized into "first phase", "second phase", and "support" institutions. First phase hospitals (ie, infectious disease hospitals) are designated to treat patients with COVID-19, in moderate, severe, and critical forms. Second phase hospitals house patients with COVID-19, when the available first phase beds are outnumbered. In the second phase and support hospitals, distinct circuits were implemented for COVID-19 patients. All staff were instructed to implement prevention and 
Table I Study Participants: Descriptive Statistics for Socio-Demographic and Professional Data

\begin{tabular}{|c|c|c|c|c|}
\hline \multirow[t]{2}{*}{ Variables } & \multirow{2}{*}{$\begin{array}{l}\text { All Respondents (Total 364) } \\
\text { N (\%) }\end{array}$} & \multirow{2}{*}{$\begin{array}{l}\text { Females (Total 29I) } \\
\mathbf{N}(\%)\end{array}$} & \multirow{2}{*}{$\begin{array}{l}\text { Males (Total 73) } \\
\mathbf{N}(\%)\end{array}$} & \multirow[t]{2}{*}{ p-value ${ }^{(a)}$} \\
\hline & & & & \\
\hline \multicolumn{5}{|l|}{ Age group } \\
\hline Less or equal 30 years & $85(23.4 \%)$ & 61 (21\%) & $24(32.9 \%)$ & $0.027^{*}$ \\
\hline $31-40$ years & $107(29.4 \%)$ & $88(30.2 \%)$ & $19(26 \%)$ & \\
\hline $4 I-50$ years & III (30.5\%) & 97 (33.3\%) & $14(19.2 \%)$ & \\
\hline Over 50 years & $6 \mathrm{I}(16.8 \%)$ & 45 (I5.5\%) & 16 (21.9\%) & \\
\hline \multicolumn{5}{|l|}{ Level of education } \\
\hline High school & $14(3.8 \%)$ & $13(4.5 \%)$ & $\mathrm{I}(\mathrm{l} .4 \%)$ & $0.04 *$ \\
\hline College & $88(24.2 \%)$ & $77(26.5 \%)$ & II (I5.1\%) & \\
\hline Higher & $262(72 \%)$ & $201(69.1 \%)$ & 61 (83.6\%) & \\
\hline \multicolumn{5}{|l|}{ Marital status } \\
\hline Single & $60(16.5 \%)$ & 44 (15.1\%) & 16 (21.9\%) & 0.402 \\
\hline Live-in relationship & $64(17.6 \%)$ & $50(17.2 \%)$ & 14 (19.2\%) & \\
\hline Married & $203(55.8 \%)$ & $164(56.4 \%)$ & 39 (53.4\%) & \\
\hline Divorced & 34 (9.3\%) & $30(10.3 \%)$ & $4(5.5 \%)$ & \\
\hline Widow/Widower & $3(0.8 \%)$ & $3(1 \%)$ & 0 & \\
\hline \multicolumn{5}{|l|}{ Current professional status } \\
\hline Consultant & $87(23.9 \%)$ & $62(21.3 \%)$ & $25(34.2 \%)$ & $0.01 *$ \\
\hline Specialty doctor & $5 \mathrm{I}(14 \%)$ & 37 (12.7\%) & 14 (19.2\%) & \\
\hline Trainee/junior doctor & 76 (20.9\%) & 58 (19.9\%) & I8 (24.7\%) & \\
\hline Senior nurse & I0I (27.7\%) & 91 (31.1\%) & $10(13.7 \%)$ & \\
\hline Trainee nurse & $25(6.9 \%)$ & $22(7.6 \%)$ & $3(4.1 \%)$ & \\
\hline Other & $24(6.6 \%)$ & $21(7.2 \%)$ & $3(4.1 \%)$ & \\
\hline \multicolumn{5}{|l|}{ Hospital work experience } \\
\hline Less or equal to I year & $51(14 \%)$ & 39 (I3.4\%) & $12(16.4 \%)$ & 0.686 \\
\hline $2-5$ years & $116(31.9 \%)$ & $93(32 \%)$ & $23(31.5 \%)$ & \\
\hline $6-10$ years & $39(10.7 \%)$ & $29(10 \%)$ & $10(13.7 \%)$ & \\
\hline $1 \mathrm{I}-15$ years & $36(9.9 \%)$ & $28(9.6 \%)$ & $8(11 \%)$ & \\
\hline Over 15 years & $122(33.5 \%)$ & $102(35.1 \%)$ & $20(27.4 \%)$ & \\
\hline Having coordination responsibilities & $98(26.9 \%)$ & $78(26.8 \%)$ & $20(27.4 \%)$ & 0.919 \\
\hline Red area activity & $55(\mid 5.1 \%)$ & $46(15.8 \%)$ & $9(12.3 \%)$ & 0.458 \\
\hline Acknowledged/perceived professional support & $275(75.5 \%)$ & $223(76.6 \%)$ & $52(71.2 \%)$ & 0.337 \\
\hline Family support received & $344(94.5 \%)$ & $274(94.2 \%)$ & $70(95.9 \%)$ & 0.561 \\
\hline \multicolumn{5}{|l|}{ In care } \\
\hline Minor child/children & $109(29.9 \%)$ & 90 (30.9\%) & $19(26 \%)$ & 0.426 \\
\hline Elderly persons & $56(15.4 \%)$ & $42(14.4 \%)$ & $14(19.2 \%)$ & \\
\hline Both minor and elderly persons & $55(15.1 \%)$ & 47 (16.2\%) & $8(11 \%)$ & \\
\hline None & $144(39.6 \%)$ & $112(38.5 \%)$ & $32(43.8 \%)$ & \\
\hline Provided ID and requested feedback & $140(38.5 \%)$ & $117(40.2 \%)$ & $23(31.5 \%)$ & 0.172 \\
\hline
\end{tabular}

Notes: ${ }^{(a)}$ Chi-square test (either asymptotic or Monte-Carlo simulation with 10,000 samples). $*$ Statistical significance, $\mathrm{p}<0.05$.

Abbreviation: $\mathrm{N}(\%)$, observed frequency (percent).

protective measures. Regular workload and organizations' working styles suffered dramatic changes, which put high pressure on healthcare workers. The burnout syndrome among Romanian healthcare workers has been identified. ${ }^{62,63}$ We aimed to evaluate the psychological distress associated with the work-related experiences of medical professionals and supporting staff during the pandemic outbreak. 
Table 2 Anxiety, Burnout, Stress, Resilience, and Empathy Among the Study Participants: Scale Measurements

\begin{tabular}{|c|c|c|c|c|}
\hline & All Respondents (Total 364) & Females (Total 29I) & Males (Total 73) & \\
\hline Scales (No of items; Cronbach's alpha) & Median (IQR) & Median (IQR & Median (IQR & $p$-value ${ }^{(a)}$ \\
\hline HARS Total $^{(\mathrm{a})}$ (14 items; 0.915) & $5(2-10)$ & $5(2-I I)$ & $6(2-10)$ & 0.789 \\
\hline Maslach Total ${ }^{(\mathrm{a})}$ (16 items; 0.928) & II $(4-24)$ & $11(5-24)$ & $12(6-24)$ & 0.525 \\
\hline Maslach A emotional ${ }^{(\mathrm{a})}$ (5 items; 0.892) & $6(2-10)$ & $6(2-10)$ & $6(2-11)$ & 0.905 \\
\hline Maslach B depersonalization ${ }^{(\mathrm{a})}$ (5 items; 0.834$)$ & $3(0-7)$ & $3(0-7)$ & $4(1-9)$ & 0.186 \\
\hline Maslach C diminishedProfEff ${ }^{(a)}(6$ items; 0.843 ) & $2(0-6)$ & $2(0-6)$ & $2(0-5)$ & 0.756 \\
\hline PHQ-9 Total ${ }^{(\mathrm{a})}$ (9 items; 0.884$)$ & $5(2-8)$ & $5(2-8)$ & $4(2-8)$ & 0.845 \\
\hline Resilience Total ${ }^{(\mathrm{a})}$ (25 items; 0.923) & $74(62.5-84)$ & $75(63.5-84.5)$ & $70(60-81)$ & $0.04 *$ \\
\hline Empathy Total $^{(\mathrm{a}) \#}(8$ items; 0.648) & $8(8-8)$ & $8(8-8)$ & $8(7-8)$ & $0.003 * *$ \\
\hline Binary variable & $N(\%)$ & $N(\%)$ & $N(\%)$ & $\mathrm{p}$-value, ${ }^{(\mathrm{b})}$ \\
\hline Empathy $^{(\mathrm{b})}$ & $282(77.5 \%)$ & 235 (80.8\%) & 47 (64.4\%) & $0.003 * *$ \\
\hline
\end{tabular}

Notes: (a) Mann-Whitney U-test (two gender groups). ${ }^{(b)}$ Chi-square test (proportions in the two gender groups). "Cronbach's alpha $<0.8$ but over 0.6 , with rather few items, and measurements' distribution strongly skewed toward the maximum value 8 - the measurements were re-coded into the binary variable Empathy. *Statistical significance, $\mathrm{p}<0.05$; **High statistical significance, $\mathrm{p}<0.01$.

Abbreviations: HARS, Hamilton Anxiety Rating Scale; IQR, inter-quartile range; N (\%), observed frequency (percent); PHQ-9, 9-item Patient Health Questionnaire; Maslach, Maslach Burnout Inventory - General Survey.

Table 3 Anxiety, Burnout, Stress, Resilience, and Empathy Across the Professional Status Categories

\begin{tabular}{|c|c|c|c|c|c|c|c|}
\hline & Consultant & Specialty Doctor & Trainee Doctor & Senior Nurse & Trainee Nurse & Other & \\
\hline & $n=87$ & $n=5$ I & $n=76$ & $n=101$ & $n=25$ & $n=24$ & \\
\hline Scales & Median (IQR) & Median (IQR) & Median (IQR) & Median (IQR) & Median (IQR) & Median (IQR) & $P$ value ${ }^{(a)}$ \\
\hline HARS Total & $5(2-9)$ & $5(3-9)$ & $7(4-13)$ & $5(1-7)$ & $5(2-12)$ & $2.5(0-11)$ & $0.011 *$ \\
\hline Maslach Total & $13(6-25.5)$ & $13(6-25.5)$ & 14 (7-29) & $9(2-18)$ & $8(3-15)$ & II (I-2I.5) & $0.014^{*}$ \\
\hline Maslach A & $6(2.5-12.5)$ & $7(3-12.5)$ & $7(2-10.5)$ & $4(I-8)$ & $5(I-9)$ & $5(0-8.5)$ & 0.058 \\
\hline Maslach B & $4(I-8)$ & $2(I-9)$ & $4.5(0-11.5)$ & $2(0-6)$ & I (0-6) & $1.5(0-6.5)$ & $0.05^{*}$ \\
\hline Maslach C & $2(0-6)$ & $3(0-6.5)$ & $4.5(I-9)$ & I (0-5) & $0(0-2)$ & I (0-6) & $0.003^{* *}$ \\
\hline PHQ-9 Total & $5(2-8)$ & $5(2.5-8)$ & $6(3-8)$ & $4(2-6)$ & $4(2-7)$ & $3(0.5-6.5)$ & $0.03^{*}$ \\
\hline Resilience Total & $71(60.5-80.5)$ & $74(63-84)$ & $70.5(60-80.5)$ & 79 (68-87) & $80(6 I-87)$ & $76.5(65.5-87)$ & $0.03 *$ \\
\hline \multicolumn{8}{|c|}{ \{HARS Total, Maslach A, Maslach B, Maslach C, PHQ-9 Total, Resilience Total\} nonparametric multivariate permutation test, $\mathrm{p}=0.048^{*}$} \\
\hline Binary variable & $N(\%)$ & $\mathrm{N}(\%)$ & $\mathrm{N}(\%)$ & $\mathrm{N}(\%)$ & $\mathrm{N}(\%)$ & $\mathrm{N}(\%)$ & $\mathrm{P}$ value, ${ }^{(\mathrm{b})}$ \\
\hline Empathy8 & 66 (75.9\%) & $43(84.3 \%)$ & 51 (67.1\%) & 86 (85.1\%) & 21 (84\%) & 15 (62.5\%) & $0.021^{*}$ \\
\hline
\end{tabular}

Notes: ${ }^{\left({ }^{a}\right)}$ Kruskal-Wallis test (six professional categories) ${ }^{(b)}$ Chi-square test (proportions in the six professional categories) $*$ Statistical significance, $\mathrm{p}<0.05 ; * *$ High statistical significance, $p<0.01$.

Abbreviations: HARS, Hamilton Anxiety Rating Scale; IQR, inter-quartile range; N (\%), observed frequency (percent); PHQ-9, 9-item Patient Health Questionnaire; Maslach, Maslach Burnout Inventory - General Survey.

Regarding psychological outcomes, we found higher resilience for female versus male healthcare workers, and coordinators versus non-coordinators. Moreover, professionals working in the frontline "red area" seemed more resilient and the trainees were the least resilient (although the differences did not reach statistical significance).
Similar findings were reported by Pollock et $\mathrm{a}^{45}$ for health and social care workers, and Zager Kocjan et $\mathrm{al}^{46}$ for the general population. Both studies also proposed policyrelated organizational solutions for enhancing the resilience and improving individuals' adaptability in difficult times. In contrast, Joana Kuntz ${ }^{64}$ and Ye et al ${ }^{65}$ chose to 
Table 4 Anxiety, Burnout, Stress, Resilience, and Empathy in Relation to Coordination Responsibilities and Red Area Activity

\begin{tabular}{|c|c|c|c|c|c|c|}
\hline & \multicolumn{2}{|c|}{ Coordination Responsibilities $^{(c)}$} & & \multicolumn{2}{|c|}{ Red Area Activity ${ }^{(d)}$} & \\
\hline & No $(n=266)$ & Yes $(n=98)$ & & No $(n=309)$ & Yes $(n=55)$ & \\
\hline Scales & Median (IQR) & Median (IQR) & $P$ value ${ }^{(a)}$ & Median (IQR) & Median (IQR) & $P$ value ${ }^{(a)}$ \\
\hline HARS Total ${ }^{(\mathrm{a})}$ & $5(2-10)$ & $5(2-10)$ & 0.952 & $5(2-10)$ & $8(3-13)$ & $0.03^{*}$ \\
\hline Maslach Total ${ }^{(\mathrm{a})}$ & II.5 (4-26) & II (6-2I) & 0.872 & II (4-22) & $15(6-29)$ & 0.078 \\
\hline Maslach $\mathrm{A}^{(\mathrm{a})}$ & $5(1-10)$ & $6(3-10)$ & 0.211 & $6(2-10)$ & $7(3-12)$ & 0.073 \\
\hline Maslach B ${ }^{(a)}$ & $3(0-8)$ & $2.5(0-6)$ & 0.205 & $3(0-7)$ & $4(I-9.5)$ & 0.12 \\
\hline Maslach $C^{(a)}$ & $2(0-7)$ & I $(0-5)$ & 0.134 & $2(0-6)$ & $3(0-9)$ & 0.088 \\
\hline PHQ-9 Total ${ }^{(a)}$ & $5(2-8)$ & $4.5(2-7)$ & 0.694 & $4(2-7)$ & $6(3-9)$ & $0.018^{*}$ \\
\hline Resilience Total $^{(\mathrm{a})}$ & $74(60-83)$ & 77 (67-88) & $0.004^{* *}$ & $74(63-84)$ & $77(59.5-84)$ & 0.877 \\
\hline \multicolumn{7}{|c|}{ (c) $\{$ HARS Total, Maslach A, Maslach B, Maslach C, PHQ-9 Total, Resilience Total\} nonparametric multivariate permutation test, $\mathrm{P}=0.066$} \\
\hline \multicolumn{7}{|c|}{ (d) $\left\{\right.$ HARS Total, Maslach A, Maslach B, Maslach C, PHQ-9 Total, Resilience Total\} nonparametric multivariate permutation test, $\mathrm{p}=0.048^{*}$} \\
\hline Binary variable & $\mathrm{N}(\%)$ & $\mathrm{N}(\%)$ & p value ${ }^{(b)}$ & $\mathrm{N}(\%)$ & $\mathrm{N}(\%)$ & P value ${ }^{(b)}$ \\
\hline Empathy $8^{(\mathrm{b})}$ & $206(77.4 \%)$ & $76(77.6 \%)$ & 0.983 & $238(77 \%)$ & $44(80 \%)$ & 0.626 \\
\hline
\end{tabular}

Notes: (a) Mann-Whitney $U$-test (two groups) ${ }^{(b)}$ Chi-square test (proportions in the two groups) ${ }^{(\mathrm{c}),(\mathrm{d})}$ Nonparametric multivariate permutation test. *Statistical significance, $\mathrm{p}<0.05$; **High statistical significance, $\mathrm{p}<0.01$.

Abbreviations: HARS, Hamilton Anxiety Rating Scale; IQR, inter-quartile range; N (\%), observed frequency (percent); PHQ-9, 9-item Patient Health Questionnaire; Maslach, Maslach Burnout Inventory - General Survey.

Table 5 Anxiety, Burnout, Stress, Resilience, and Empathy in Relation to the Perceived Support from the Professional Environment or the Family, Friends, and Acquaintances

\begin{tabular}{|c|c|c|c|c|c|c|}
\hline & \multicolumn{2}{|c|}{ Professional Support ${ }^{(c)}$} & & \multicolumn{2}{|c|}{ Family Support } & \multirow[t]{2}{*}{ p value ${ }^{(a),(b)}$} \\
\hline & No $(n=89)$ & Yes $(n=275)$ & & No $(n=20)$ & Yes $(n=344)$ & \\
\hline Scales & Median (IQR) & Median (IQR) & $P$ value ${ }^{(a)}$ & Median (IQR) & Median (IQR) & \\
\hline HARS Total ${ }^{(\mathrm{a})}$ & $7(3-14)$ & $4(1.5-9.5)$ & $<0.001 * *$ & $7.5(4-16)$ & $5(2-10)$ & 0.085 \\
\hline Maslach Total ${ }^{(\mathrm{a})}$ & 22 9-35) & $9(4-18.5)$ & $<0.001 * *$ & I $4.5(\mid-25.5)$ & II (5-23.5) & 0.907 \\
\hline Maslach $A^{(a)}$ & $8(4-16)$ & $5(I-9)$ & $<0.00 I^{* *}$ & $6(1-13)$ & $6(2-10)$ & 0.687 \\
\hline Maslach B ${ }^{(a)}$ & $7(2-14)$ & $2(0-6)$ & $<0.001^{* *}$ & $3(0-6.5)$ & $3(0-7)$ & 0.661 \\
\hline Maslach $C^{(a)}$ & $5(0-10)$ & I $(0-5)$ & $<0.00 I^{* *}$ & $2(0-6.5)$ & $2(0-6)$ & 0.78 \\
\hline PHQ-9 Total ${ }^{(a)}$ & $7(3-10)$ & $4(2-7)$ & $<0.00 I^{* *}$ & $5.5(3-11.5)$ & $4(2-8)$ & 0.182 \\
\hline Resilience Total $^{(\mathrm{a})}$ & $68(60-80)$ & $75(63-85)$ & $0.009 * *$ & $69(55.5-83.5)$ & $74.5(63-84)$ & 0.353 \\
\hline \multicolumn{7}{|c|}{ (c) $\{$ HARS Total, Maslach A, Maslach B, Maslach C, PHQ-9 Total, Resilience Total\} nonparametric multivariate permutation test, $\mathrm{p}<0.00 \mathrm{I} * *$} \\
\hline Binary variable & N (\%) & $\mathrm{N}(\%)$ & P value ${ }^{(b)}$ & $\mathrm{N}(\%)$ & N (\%) & P value ${ }^{(b)}$ \\
\hline Empathy $8^{(b)}$ & $61(68.5 \%)$ & $221(80.4 \%)$ & $0.02 * *$ & $18(90 \%)$ & $264(76.7 \%)$ & 0.269 \\
\hline
\end{tabular}

Notes: ${ }^{(a)}$ Mann-Whitney U-test (two groups) ${ }^{(b)}$ Chi-square test (proportions in the two groups) ${ }^{(c)}$ Nonparametric multivariate permutation test $*$ Statistical significance, $\mathrm{p}<$ 0.05 ; **High statistical significance, $p<0.01$.

Abbreviations: HARS, Hamilton Anxiety Rating Scale; IQR, inter-quartile range; N (\%), observed frequency (percent); PHQ-9, 9-item Patient Health Questionnaire; Maslach, Maslach Burnout Inventory - General Survey.

focus their research on individuals' characteristics and identify psychosocial risk factors. Interestingly for our study, the respondents who chose to provide personal contact information and requested feedback proved to be more resilient, but no other distinguishable patterns were found. 
Table 6 The Logistic Regression Model for Empathy. A Step-Wise Analysis Was Conducted, Based on the Akaike Information Criterion: For Models I, 2, and 3, Only Variables That Remained in the Model are Shown. In Model I, The Professional Support Was Statistically Significant, Almost Doubling the Odds of Empathy. In Model 3, The Effect of Professional Support Became Less Relevant for the Empathy and the Increase in Burnout-Depersonalization Accounted for the Loss of Empathy, with High Statistical Significance. Exp (B) is Equivalent to the Odds Ratio (OR), a Measure of Relationship's Strength Between the Predictors and Binary Empathy8 Outcome

\begin{tabular}{|c|c|c|c|}
\hline Predictor & $B \pm$ Std. err & p-value & $\operatorname{Exp}(B)(95 \% \mathrm{Cl})$ \\
\hline GenderM & $-0.892 \pm 0.305$ & $0.003 * *$ & $0.410(0.225-0.745)$ \\
\hline Marital status & $-0.014 \pm 0.142$ & 0.919 & NA \\
\hline Professional status & $-0.051 \pm 0.086$ & 0.556 & NA \\
\hline \multicolumn{4}{|c|}{$\mathrm{AlC}_{0}=387.75,4 \mathrm{df} ;$ Nagelkerke R-square $=0.104$} \\
\hline \multicolumn{4}{|c|}{ Model I: Empathy8 GenderM + Professional support Controlling for: Marital status and Professional status } \\
\hline Predictor & $B \pm$ Std. err & p-value & $\operatorname{Exp}(B)(95 \% \mathrm{Cl})$ \\
\hline GenderM & $-0.887 \pm 0.306$ & $0.003 * *$ & $0.412(0.226-0.75 I)$ \\
\hline Professional support & $0.631 \pm 0.288$ & $0.028^{*}$ & $1.88(1.069-3.305)$ \\
\hline \multicolumn{4}{|c|}{$\mathrm{AIC}_{1}=384.87,5 \mathrm{df} ;\left(\mathrm{AIC}_{1}, \mathrm{AIC}_{0}\right), \mathrm{p}=0.027 * ;$ Nagelkerke R-square $=0.122$} \\
\hline \multicolumn{4}{|c|}{ Model 2: Empathy8 GenderM + Professional support + Resilience Total Controlling for: Marital status and Professional status } \\
\hline Predictor & $B \pm$ Std. err & $\mathrm{p}$-value & $\operatorname{Exp}(B)(95 \% \mathrm{Cl})$ \\
\hline GenderM & $-0.845 \pm 0.309$ & $0.006 * *$ & $0.43(0.234-0.787)$ \\
\hline Professional support & $0.5521 \pm 0.292$ & 0.058 & $1.737(0.98 \mathrm{I}-3.078)$ \\
\hline Resilience Total & $0.022 \pm 0.009$ & $0.016^{*}$ & $1.023(1.004-1.04 I)$ \\
\hline \multicolumn{4}{|c|}{$\left.\mathrm{AlC}_{2}=380.39,6 \mathrm{df} ;\left(\mathrm{AlC}_{2}, \mathrm{AIC}\right)_{1}\right), \mathrm{P}=0.0 \mathrm{II} * ;$ Nagelkerke R-square $=0.144$} \\
\hline \multicolumn{4}{|c|}{$\begin{array}{l}\text { Model 3: Empathy8 GenderM + Professional support + Resilience Total + Maslach B depersonalization Controlling for: Marital status ar } \\
\text { Professional status }\end{array}$} \\
\hline Predictor & $B \pm$ Std. err & $\mathrm{p}$-value & $\operatorname{Exp}(B)(95 \% \mathrm{Cl})$ \\
\hline GenderM & $-0.817 \pm 0.319$ & $0.009 * *$ & $0.442(0.237-0.826)$ \\
\hline Professional support & $0.206 \pm 0.32$ & 0.582 & $1.228(0.656-2.3)$ \\
\hline Resilience Total & $0.015 \pm 0.01$ & 0.136 & $1.015(0.995-1.034)$ \\
\hline Maslach B depersonalization & $-0.070 \pm 0.023$ & $<0.00 I^{* *}$ & $0.926(0.885-0.969)$ \\
\hline
\end{tabular}

Notes: *Statistical significance, $\mathrm{p}<0.05$; **High statistical significance, $\mathrm{p}<0.01$.

Abbreviations: AIC, Akaike information criterion; $\mathrm{Cl}$, confidence interval; df, degrees of freedom; NA, not applicable.

Although the PHQ-9 scores were not particularly high in this survey sample, a rather slight depression was reported, with increased levels among the frontline workers. Physicians seemed more depressed than nurses, with young doctors being the most affected. Moreover, trainee doctors reported a heavy burden of anxiety and a lower level of empathy. Research conducted to date has presented contradicting results regarding these aspects, although most articles reported high levels of depression and/or anxiety, and overall psychological problems for healthcare workers during this pandemic, ${ }^{66-68}$ which is similar to other reports on mental health risks during disasters in the past. ${ }^{69}$

The individuals' experience of burnout was significantly heavier among physicians compared to nurses, and higher for workers on the frontline. Burnout among 




Figure I The triad-type relationship between perceived professional support, burnout-depersonalization, and empathy. The boxes are proportional to the inter-quartile range (IQR) with medians marked in-between, and the whiskers are proportional to I.5*IQR (or trimmed to the minimum or maximum values). The bullets and stars are outliers and extreme values, respectively.

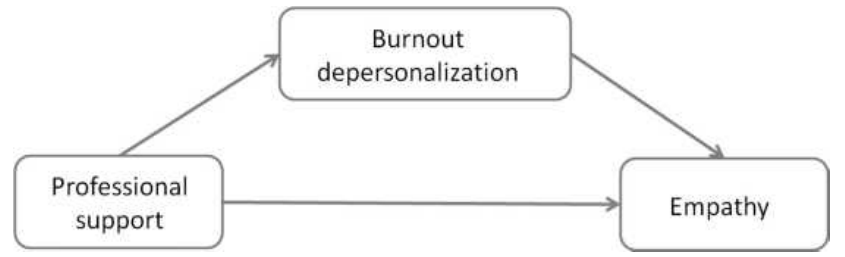

Figure 2 Causal mediation model for the role of burnout-depersonalization on the association between the professional support and the empathy of healthcare professionals: two causal paths feed into the outcome variable (ie the empathy).

physicians has been acknowledged as a global problem. ${ }^{33,35,36}$ Increased burnout has also been reported in Romanian medical staff after the start of this pandemic, with even higher levels in trainees. ${ }^{62}$ Different protective factors against burnout, such as training, continuing education, supervision, recognition, and continuous feedback were underlined in these specific circumstances of the COVID-19 pandemic in Romania. ${ }^{63}$
For all respondents, perceived family support seemed to play a relieving role, but did not have a significant impact. On the other hand, the reported professional support made an important and significant difference across all the psychological measurements (ie, anxiety, depression, burnout, and resilience). The missing professional support was also decisive for the missing empathy. The relationship between burnout and empathy has been assiduously investigated. $^{18,34,40,70,71}$ In our study, female professionals seemed by far more empathetic than their male co-workers (80.8\% versus $64.4 \%)$. Trainee doctors seemed to be the least empathetic, an issue that could be related to their higher levels of depression and anxiety, and lower levels of resilience. It has been suggested that women are more empathetic than their male peers. ${ }^{72,73}$ A possible explanation might be that women are, biologically, more inclined to manifest empathy and assume a caregiver's role. ${ }^{74-76}$ 
Table 7 Direct and Causal Mediation Effects of Professional Support on the Empathy, with Burnout-Depersonalization as the Mediator. Bias Corrected and Accelerated (BCa) Method of Bootstrap Re-Sampling (1000 Samples) Was Applied to Determine the 95\% Confidence Intervals

\begin{tabular}{|c|c|c|}
\hline Effect & Effect Estimate on Empathy $(95 \% \mathrm{Cl})$ & p-value \\
\hline ACME (no Professional support) & $0.080 I(0.0304-0.15)$ & $0.002 * *$ \\
\hline ACME (with Professional support) & $0.0396(-0.0083-0.09)$ & 0.11 \\
\hline ADE (no Professional support) & $0.0498(-0.0646-0.17)$ & 0.37 \\
\hline ADE (with Professional support) & $0.0093(-0.0911-0.13)$ & 0.83 \\
\hline Total Effect & $0.0894(-0.0234-0.21)$ & 0.094 \\
\hline ACME (average) & $0.0599(0.0238-0.10)$ & $<0.001^{* *}$ \\
\hline ADE (average) & $0.0295(-0.0774-0.15)$ & 0.536 \\
\hline \multicolumn{3}{|c|}{ Testing the interaction independent variable $\leftrightarrow$ mediator } \\
\hline $\operatorname{ACME}(I)-\operatorname{ACME}(0)$ & $-0.0405(-0.1158-0.0149)$ & 0.176 \\
\hline
\end{tabular}

Note: **High statistical significance, $\mathrm{p}<0.01$.

Abbreviations: ACME, average causal mediation effects; $\mathrm{ADE}$, average direct effects; $\mathrm{Cl}$, confidence interval.

The constructed regression model proved the high odds towards empathy among women and the significant loss of empathy related to the depersonalization component of the burnout experience. The regression analysis we conducted also brought forward the role of professional support in sustaining empathy towards others (either patients or coworkers), regardless of the individuals' personality dimensions or characteristics, such as professional or marital status, or even their resilience.

The causal direction in the relationship between burnout and empathy has been a matter of debate and contradictory associations have been reported. ${ }^{34,40,71}$ Wilkinson et $\mathrm{al}^{18}$ provided consistent evidence for a negative association between burnout and empathy, acknowledging them both as fundamental contributory elements to the quality of healthcare. Team-based models of care and educational solutions have been proposed for preventing the burnout and addressing the cognitive dimension of empathy. ${ }^{33,34,47}$ Empathy should be seen not only as a trait, but as a dynamic factor that can be improved by indirect measures aimed at reducing the burnout or improving the work environment, and implementing a work culture centered on human wellbeing, of employees and patients alike. Measuring empathy might provide additional information regarding the implement of such change.

We propose a mediation model for the relationship of professional support - burnout - empathy, based on the role-shifting of burnout from effect to cause, depending on the focus of the analysis. The model in this applied research arguably clarifies the decisive role of professional support in preventing the burnout and the subsequent loss of empathy. The mediator would explain how external physical facts or events take on internal psychological significance.

A lack of social support has been reported as related to the emergence of psychiatric disorders during this pandemic. ${ }^{77}$ Similarly, a positive work environment, trusted leadership, and helpful professional relationships had been considered to be protective elements against the burnout. $^{78}$ These reports would confirm our findings. Burnout and depersonalization are recognized as being related to anxiety, stress, and other psychiatric disorders. ${ }^{78-82}$ Likewise, empathy is connected with professional efficacy and burnout. ${ }^{18,83}$ The model we propose is in concordance with existing information about systemic interrelations in the workplace. The quality of the healthcare system depends on the wellbeing of professional providers. ${ }^{84-86}$ Supportive leadership is an important factor towards workplace wellbeing, and thus the perceived professional support is acknowledged. ${ }^{87}$ Solutions for managing or mitigating the burnout are neither easy nor quick. ${ }^{88}$ Although it has been accepted as exacerbating the stress and individuals' inability to recover, there are difficulties in defining, diagnosing, and addressing its underlying structural causes. ${ }^{88,89}$

To sum up, we explored and evaluated the relationship between healthcare workers' vulnerability (with a highlight on gender differences, professional roles and responsibilities) 


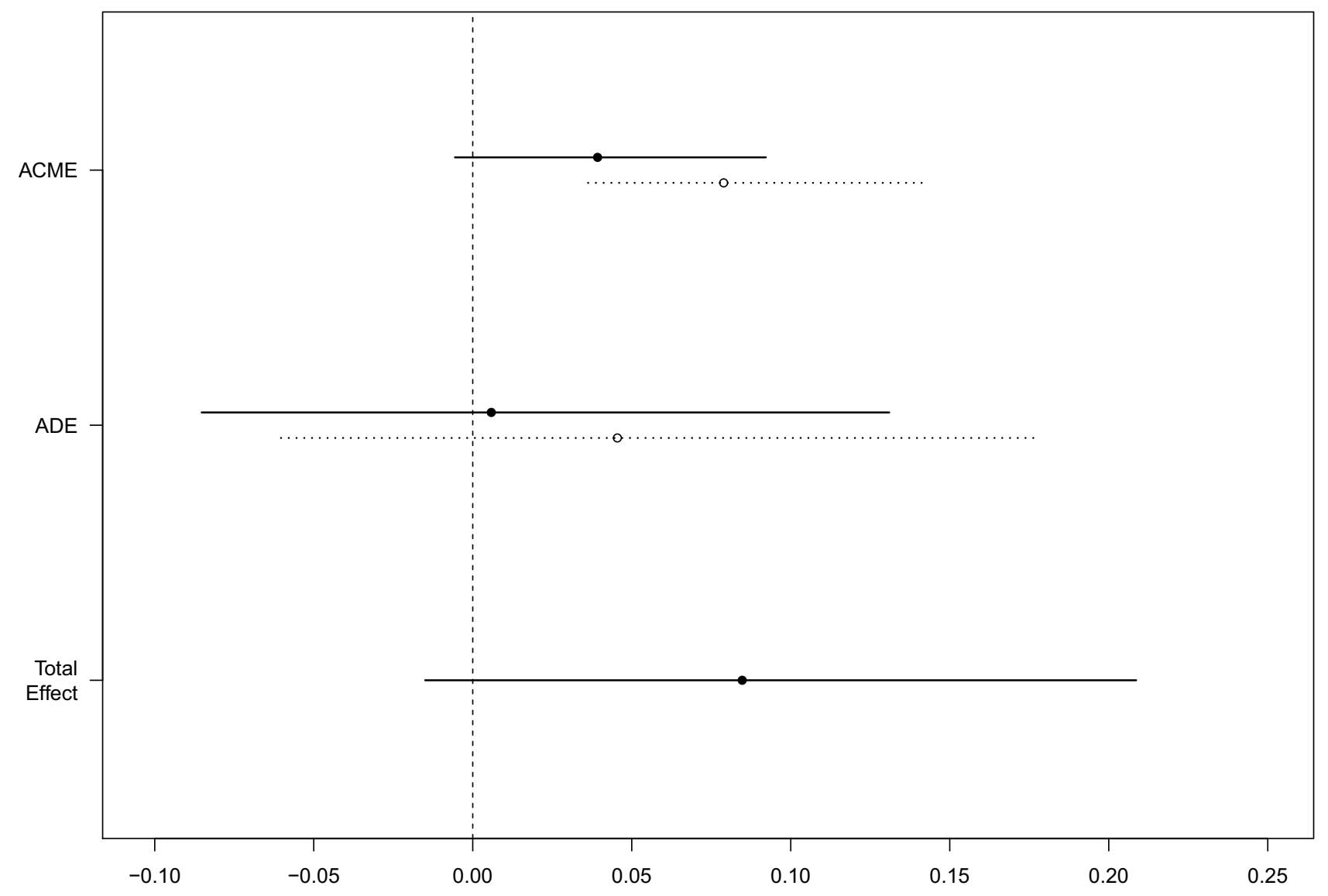

Figure 3 Direct and causal mediation effects of professional support on the empathy. Abbreviations: ACME, average causal mediation effects; ADE, average direct effects. Each estimate value is shown with bullets, with the $95 \%$ confidence intervals as horizontal lines. Intervals for the situation with no perceived professional support are shown in dotted lines. The no effect reference is shown as a vertical dotted line.

and resilience factors, when confronted with stress caused by the onset of the COVID-19 pandemic. The lack of support in the professional environment was associated with higher anxiety and stress, less resilience and empathy, and higher burnout scores. A triad-type relationship between professional support, burnout-depersonalization, and lack of empathy was observed and analyzed through a mediation mechanism. The missing professional support for medical staff might generate major vulnerabilities in the relationship between healthcare workers and their patients, and unfavorable mid- and long-term consequences on the quality of healthcare services.

\section{Limitations of the Study}

The main limitation of this study was that a crosssectional design cannot prove causality. However, the analytic procedure employed for testing and measuring the mediation role of burnout allowed us to bring evidence toward designing future longitudinal/interventional studies.
Other limitations were generated by the assumptions for employing multiple regression to estimate the mediation model: (a) the dependent variable does not cause the mediator; (b) there is no measurement error in the mediator. The first assumption was met, but the self-reported burnout scores might entail unavoidable measurement errors, with an attenuation effect on the size of association measures and subsequent overestimation bias of the independent variable (up to the extent that it would cause the mediator). Consequently, as a psychological variable, it would tend to produce an underestimate of the mediator's effect and even overlook successful mediators. A possible solution might be to test the linkages by employing structural modeling, but this approach would go beyond the scope of this applied research. Burnout was not actually proved as a necessary condition for the effect of professional support to occur on empathy; on the other hand, the small and non-significant direct effect (although not zero) suggests a robust and generalizable mediation model, in spite of the above mentioned limitations. 
Additional caveats were generated by the auto-selection of individuals participating in the survey (the respondents might have been more enthusiastic or willing to engage in their work, and thus might have had higher levels of resilience or empathy, compared to non-respondents). The potential misleading effect of the outliers on the scales' scorings was compensated by employing non-parametric statistical methods in data analysis.

In addition to the inherent errors related to selfreporting, the coding of empathy and professional support as dichotomies is error-prone as well. However, many other recent studies employed similar coding strategies, since the number of measurements is inherently limited in most study designs.

\section{Conclusion}

We brought evidence towards a mediation role of burnout in the relationship between the organizational support and the empathy among healthcare workers during the COVID-19 outbreak. This viewpoint is a systemic one, offering perspectives over several factors involved. The circularity of the approach confers indirect suggestions as to how interventions may be designed.

Health policies would seek workable solutions to address modifiable factors to alleviate the burnout and implement the lessons beyond the current pandemic, and to embody compassionate and patient-centered organizational culture. We suggest that implementing prevention programs aimed at relieving the pressure on healthcare personnel outside the range of the received training or confidence in their own professionalism would avoid the burnout and its toll of empathy, thus evading subsequent degradation of medical services' quality. Such programs might include mental health screening for identifying early signs of burnout, psychological support and comprehensive educational interventions for mid-career professionals, residents, and medical students.

\section{Data Sharing Statement}

Data sets are not publicly available but de-identified data may be provided upon request.

\section{Acknowledgments}

The authors kindly acknowledge the colleagues who volunteered to participate in this study and share their data to further our understanding of work-related stress.

\section{Disclosure}

The authors report no conflicts of interest in this work.

\section{References}

1. World Health Organization [page on the Internet]. Director-General's opening remarks at the media briefing on COVID-19; 2020. Available from: https://www.who.int/emergencies/diseases/novel-cor onavirus-2019. Accessed January 3, 2021.

2. Zhang J, Lu H, Zeng H, et al. The differential psychological distress of populations affected by the COVID-19 pandemic. Brain Behav Immun. 2020;87:49-50. doi:10.1016/j.bbi.2020.04.031

3. Cai W, Lian B, Song X, Hou T, Deng G, Li H. A cross-sectional study on mental health among health care workers during the outbreak of Corona Virus Disease 2019. Asian J Psychiatr. 2020;51:102111. doi:10.1016/j.ajp.2020.102111

4. Lai J, Ma S, Wang Y, et al. Factors associated with mental health outcomes among health care workers exposed to coronavirus disease 2019. JAMA Netw Open. 2020;3(3):e203976. doi:10.1001/ jamanetworkopen.2020.3976

5. Morgantini LA, Naha U, Wang $\mathrm{H}$, et al. Factors contributing to healthcare professional burnout during the COVID-19 pandemic: a rapid turnaround global survey. PLoS One. 2020;15(9):e0238217. doi:10.1371/journal.pone.0238217

6. Liao Q, Yuan J, Dong M, Yang L, Fielding R, Lam WWT. Public engagement and government responsiveness in the communications about COVID-19 during the early epidemic stage in china: infodemiology study on social media data. J Med Internet Res. 2020;22(5): e18796. doi:10.2196/18796

7. Folkman S. Stress: appraisal and coping. In: Gellman MD, Turner JR, editors. Encyclopedia of Behavioral Medicine. New York: Springer; 2013:1913-1915.

8. Coomber S, Todd C, Park G, Baxter P, Firth-Cozens J, Shore S. Stress in UK intensive care unit doctors. Br J Anaesth. 2002;89 (6):873-881. doi:10.1093/bja/aef273

9. Nussbaum M. Compassion: the basic social emotion. Soc Philos Policy. 1996;13(1):27-58. doi:10.1017/S0265052500001515

10. Weinberg A, Creed F. Stress and psychiatric disorder in healthcare professionals and hospital staff. Lancet. 2000;355(9203):533-537. doi:10.1016/S0140-6736(99)07366-3

11. McManus IC, Winder BC, Gordon D. The causal links between stress and burnout in a longitudinal study of UK doctors. Lancet. 2002;359 (9323):2089-2090. doi:10.1016/S0140-6736(02)08915-8

12. Ramirez AJ, Graham J, Richards MA, Gregory WM, Cull A. Mental health of hospital consultants: the effects of stress and satisfaction at work. Lancet. 1996;347(9003):724-728. doi:10.1016/S01406736(96)90077-X

13. Visser MR, Smets EM, Oort FJ, De Haes HC. Stress, satisfaction and burnout among Dutch medical specialists. Cmaj. 2003;168 (3):271-275.

14. Su JA, Weng HH, Tsang HY, Wu JL. Mental health and quality of life among doctors, nurses and other hospital staff. Stress and Health. J Int Soc Invest Stress. 2009;25(5):423-430. doi:10.1002/smi.1261

15. Charon R. Narrative medicine: a model for empathy, reflection, profession, and trust. JAMA. 2001;286(15):1897-1902. doi:10.1001/ jama.286.15.1897

16. Halpern J. From Detached Concern to Empathy: Humanizing Medical Practice. Oxford University Press; 2001.

17. Goetz JL, Keltner D, Simon-Thomas E. Compassion: an evolutionary analysis and empirical review. Psychol Bull. 2010;136(3):351. doi:10.1037/a0018807

18. Wilkinson H, Whittington R, Perry L, Eames C. Examining the relationship between burnout and empathy in healthcare professionals: a systematic review. Burnout Res. 2017;6:18-29. doi:10.1016/j.burn.2017.06.003

19. Chin JJ. Doctor-patient relationship: a covenant of trust. Singapore Med J. 2001;42(12):579-581.

20. O'Neill O. Autonomy and Trust in Bioethics. Cambridge University Press; 2002. 
21. Mechanic D. Changing medical organization and the erosion of trust Milbank Q. 1996;1:171-189. doi:10.2307/3350245

22. Halpern J. What is clinical empathy? J Gen Intern Med. 2003;18 (8):670-674. doi:10.1046/j.1525-1497.2003.21017.x

23. Kelley JM, Kraft-Todd G, Schapira L, Kossowsky J, Riess H, Timmer A. The influence of the patient-clinician relationship on healthcare outcomes: a systematic review and meta-analysis of randomized controlled trials. PLoS One. 2014;9(4):e94207. doi:10.1371/ journal.pone.0094207

24. Joffe S, Manocchia M, Weeks JC, Cleary PD. What do patients value in their hospital care? An empirical perspective on autonomy centred bioethics. J Med Ethics. 2003;29(2):103-108. doi:10.1136/ jme.29.2.103

25. Figley CR. Compassion fatigue: psychotherapists' chronic lack of self care. J Clin Psychol. 2002;58(11):1433-1441. doi:10.1002/jclp.10090

26. Ferri P, Guerra E, Marcheselli L, Cunico L, Di Lorenzo R. Empathy and burnout: an analytic cross-sectional study among nurses and nursing students. Acta Biomed Health Professions. 2015;86:104-115.

27. Rothschild B. Help for the Helper: The Psychophysiology of Compassion Fatigue and Vicarious Trauma. New York: WW Norton and Company; 2006.

28. Freudenberger HJ. Staff burn-out. J Soc Issues. 1974;30(1):159-165. doi:10.1111/j.1540-4560.1974.tb00706.x

29. Popa CO, Nirestean A, Ile L, Ardelean M, Moica T, Buicu G. Community mental health services - the treatment of anxiety and its effects from the point of view of the association of personality dimensions. Revista De Cercetare Si Interventie Sociala. 2013;49-60.

30. Adriaenssens J, De Gucht V, Maes S. Determinants and prevalence of burnout in emergency nurses: a systematic review of 25 years of research. Int $J$ Nurs Stud. 2015;52(2):649-661. doi:10.1016/j. ijnurstu.2014.11.004

31. Maslach C, Jackson SE. The measurement of experienced burnout. J Organ Behav. 1981;2(2):99-113. doi:10.1002/job.4030020205

32. Maslach C, Leiter MP. Understanding the burnout experience: recent research and its implications for psychiatry. World Psychiatry. 2016;15(2):103-111. doi:10.1002/wps.20311

33. Maslach C, Leiter MP. New insights into burnout and health care: strategies for improving civility and alleviating burnout. Med Teach. 2017;39(2):160-163. doi:10.1080/0142159X.2016.1248918

34. Samra R. Empathy and burnout in medicine-acknowledging risks and opportunities. J Gen Intern Med. 2018;33(7):991-993. doi:10.1007/ s11606-018-4443-5

35. The Lancet. Physician burnout: a global crisis. Lancet. 2019;394 (10193):93. doi:10.1016/S0140-6736(19)31573-9

36. Bhatnagar G. Physician burnout. Lancet. 2020;395(10221):333. doi:10.1016/S0140-6736(19)32612-1

37. Song Y, Jia Y, Sznajder K, Ding J, Yang X. Recovery experiences mediate the effect of burnout on life satisfaction among Chinese physicians: a structural equation modeling analysis. Int Arch Occup Environ Health. 2020;94:31-41. doi:10.1007/s00420-020-01554-1

38. Picard J, Catu-Pinault A, Boujut E, Botella M, Jaury P, Zenasni F. Burnout, empathy and their relationships: a qualitative study with residents in general medicine. Psychol Health Med. 2016;21 (3):354-361. doi:10.1080/13548506.2015.1054407

39. Zenasni F, Boujut E, Woerner A, Sultan S. Burnout and empathy in primary care: three hypotheses. Br J General Pract. 2012;62 (600):346-347. doi:10.3399/bjgp12X652193

40. Correia I, Almeida AE. Organizational justice, professional identification, empathy, and meaningful work during COVID-19 pandemic: are they burnout protectors in physicians and nurses? Front Psychol. 2020;11:566139. doi:10.3389/fpsyg.2020.566139

41. Mercer SW, Reynolds WJ. Empathy and quality of care. Br J General Pract. 2002;52(Suppl):S9-S12.

42. Hoffman ML. Empathy and Moral Development: Implications for Caring and Justice. Cambridge University Press; 2001.
43. Eisenberger R, Stinglhamber F. Perceived Organizational Support: Fostering Enthusiastic and Productive Employees. Washington, DC: American Psychological Association; 2011.

44. Hur WM, Han SJ, Yoo JJ, Moon TW. The moderating role of perceived organizational support on the relationship between emotional labor and job-related outcomes. Manage Decision. 2015;53 (3):605-624. doi:10.1108/MD-07-2013-0379

45. Pollock A, Campbell P, Cheyne J, et al. Interventions to support the resilience and mental health of frontline health and social care professionals during and after a disease outbreak, epidemic or pandemic: a mixed methods systematic review. Cochrane Database Syst Rev. 2020;11:CD013779. doi:10.1002/14651858.CD013779

46. Zager Kocjan G, Kavčič T, Avsec A. Resilience matters: explaining the association between personality and psychological functioning during the COVID-19 pandemic. Int J Clin Health Psychol. 2021;21(1):100198. doi:10.1016/j.ijchp.2020.08.002

47. American Medical Association [page on the Internet]. STEPS forward. Available from: https://edhub.ama-assn.org/steps-forward /module/2702509. Accessed January 3, 2021.

48. Hamilton M. Diagnosis and rating of anxiety. Br J Psychiatry. 1969;3 (special issue):76-79.

49. Kroenke K, Spitzer RL, Williams JB, The PHQ-9. validity of a brief depression severity measure. J Gen Intern Med. 2001;16(9):606-613. doi:10.1046/j.1525-1497.2001.016009606.x

50. Lupascu N, Timar B, Albai A, Roman D, Potre O, Timar R. Validation and cross-cultural adaptation of the depression Patient's Health Questionnaire-9 in the Romanian population of patients with Type 2 Diabetes Mellitus. Diabetes, Metab Syn Obes Targets Ther. 2019;12:841. doi:10.2147/DMSO.S203099

51. Bria M, Spanu F, Baban A, Dumitrascu DL. Maslach burnout inventory-general survey: factorial validity and invariance among Romanian healthcare professionals. Burnout Res. 2014;1 (3):103-111. doi:10.1016/j.burn.2014.09.001

52. Connor KM, Davidson JR. Development of a new resilience scale: the Connor-Davidson resilience scale (CD-RISC). Depress Anxiety. 2003;18(2):76-82. doi:10.1002/da.10113

53. Nussbaum L, Ogodescu A, Hogea L, Nussbaum L, Zetu I. Risk factors and resilience in the offspring of psychotic parents. Revista De Cercetare Si Interventie Sociala. 2017;56:114-122.

54. McCord DM. The International Personality Item Pool In: ZeiglerHill V, Shackelford T, editors. Encyclopedia of Personality and Individual Differences. Cham: Springer; 2017.

55. Liescu D, Popa M, Dimache R.Adaptarea romaneasca a setului international de itemi de personalitate: IPIP-Ro [Romanian adaptation of the International International Personality Item Pool: IPIP-Ro]. Psihologia Resurselor Umane. 2015;13(1):83-112.

56. Altman DG. Practical Statistics for Medical Research. London: Chapman and Hall; 1991.

57. Baron RM, Kenny DA. The moderator-mediator variable distinction in social psychological research: conceptual, strategic, and statistical considerations. J Pers Soc Psychol. 1986;51(6):1173. doi:10.1037/ 0022-3514.51.6.1173

58. MacKinnon DP, Lockwood CM, Hoffman JM, West SG, Sheets V. A comparison of methods to test mediation and other intervening variable effects. Psychol Methods. 2002;7(1):83. doi:10.1037/1082-989X.7.1.83

59. Preacher KJ, Hayes AF. Asymptotic and resampling strategies for assessing and comparing indirect effects in multiple mediator models. Behav Res Methods. 2008;40(3):879-891. doi:10.3758/BRM.40.3.879

60. Imai K, Keele L, Tingley D, Yamamoto T. Unpacking the black box of causality: learning about causal mechanisms from experimental and observational studies. Am Political Sci Rev. 2011;105:765-789. doi:10.1017/S0003055411000414

61. Cronbach LJ, Shavelson RJ. My current thoughts on coefficient alpha and successor procedures. Educ Psychol Meas. 2004;64(3):391-418. doi:10.1177/0013164404266386 
62. Dimitriu MCT, Pantea-Stoian A, Smaranda AC, et al. Burnout syndrome in Romanian medical residents in time of the COVID-19 pandemic. Med Hypotheses. 2020;144:109972. doi:10.1016/j. mehy.2020.109972

63. Cotel A E, Golu F, Pantea-Stoian A, et al. Predictors of burnout in healthcare workers during the COVID-19 pandemic. Healthcare (Basel). 2021;9(3):304. doi:10.3390/healthcare9030304

64. Kuntz JC. Resilience in times of global pandemic: steering recovery and thriving trajectories. Appl Psychol. 2020. doi:10.1111/apps.12296

65. Ye B, Zhou X, Im H, Liu M, Wang XQ, Yang Q. Epidemic rumination and resilience on college students' depressive symptoms during the COVID-19 pandemic: the mediating role of fatigue. Front Public Health. 2020;8:560983. doi:10.3389/fpubh.2020.560983

66. Spoorthy MS, Pratapa SK, Mahant S. Mental health problems faced by healthcare workers due to the COVID-19 pandemic-A review. Asian J Psychiatr. 2020;51:102119. doi:10.1016/j.ajp.2020.102119

67. Shi L, Lu ZA, Que JY, et al. Prevalence of and risk factors associated with mental health symptoms among the general population in China during the coronavirus disease 2019 pandemic. JAMA Network Open. 2020;3(7):e2014053. doi:10.1001/jamanetworkopen.2020.14053

68. Huang Y, Zhao N. Generalized anxiety disorder, depressive symptoms and sleep quality during COVID-19 outbreak in China: a web-based cross-sectional survey. Psychiatry Res. 2020;112954. doi:10.1016/j.psychres.2020.112954

69. Naushad VA, Bierens JJ, Nishan KP, et al. A systematic review of the impact of disaster on the mental health of medical responders. Prehosp Disaster Med. 2019;34(6):632-643. doi:10.1017/ S1049023X19004874

70. West CP, Dyrbye LN, Shanafelt TD. Physician burnout: contributors, consequences and solutions. J Intern Med. 2018;283(6):516-529. doi:10.1111/joim.12752

71. Kitson A, Chirico A, Gaggioli A, Riecke BE. A review on research and evaluation methods for investigating self-transcendence. Front Psychol. 2020;11:547687. doi:10.3389/fpsyg.2020.547687

72. Gault BA, Sabini J. The roles of empathy, anger, and gender in predicting attitudes toward punitive, reparative, and preventative public policies. Cogn Emot. 2000;14(4):495-520. doi:10.1080/ 026999300402772

73. Schieman S, Van Gundy K. The personal and social links between age and self-reported empathy. Soc Psychol Q. 2000;63:152. doi: $10.2307 / 2695889$

74. Van Honk J, Schutter DJ, Bos PA, Kruijt AW, Lentjes EG, BaronCohen S. Testosterone administration impairs cognitive empathy in women depending on second-to-fourth digit ratio. Proc Nat Acad Sci. 2011;108(8):3448-3452. doi:10.1073/pnas.1011891108

75. Babcock L, Recalde MP, Vesterlund L. [page on the Internet]. Why women volunteer for tasks that don't lead to promotions. Harv Bus Rev. 2018.

76. Ory MG, Hoffman III RR, Yee JL, Tennstedt S, Schulz R. Prevalence and impact of caregiving: a detailed comparison between dementia and nondementia caregivers. Gerontologist. 1999;39(2):177-186. doi:10.1093/geront/39.2.177
77. Xiao H, Zhang Y, Kong D, Li S, Yang N. The effects of social support on sleep quality of medical staff treating patients with coronavirus disease 2019 (COVID-19) in January and February 2020 in China. Med Sci Monitor Int Med J Exp Clin Res. 2020;26:e923549-1. doi:10.12659/MSM.923549

78. Rama-Maceiras P, Jokinen J, Kranke P. Stress and burnout in anaesthesia: a real world problem? Current Opinion Anesthesiol. 2015;28 (2):151-158. doi:10.1097/ACO.0000000000000169

79. Koutsimani P, Montgomery A, Georganta K. The relationship between burnout, depression, and anxiety: a systematic review and meta-analysis. Front Psychol. 2019;10:284. doi:10.3389/ fpsyg.2019.00284

80. Vela-Bueno A, Moreno-Jiménez B, Rodríguez-Muñoz A, et al. Insomnia and sleep quality among primary care physicians with low and high burnout levels. J Psychosom Res. 2008;64 (4):435-442. doi:10.1016/j.jpsychores.2007.10.014

81. Hakanen JJ, Schaufeli WB. Do burnout and work engagement predict depressive symptoms and life satisfaction? A three-wave seven-year prospective study. $J$ Affect Disord. 2012;141(2-3):415-424. doi:10.1016/j.jad.2012.02.043

82. Hakanen JJ, Schaufeli WB, Ahola K. The Job Demands-Resources model: a three-year cross-lagged study of burnout, depression, commitment, and work engagement. Work Stress. 2008;22(3):224-241. doi:10.1080/02678370802379432

83. Hojat M, Gonnella JS, Nasca TJ, Mangione S, Vergare M, Magee M. Physician empathy: definition, components, measurement, and relationship to gender and specialty. Am $J$ Psychiatry. 2002;159 (9):1563-1569. doi:10.1176/appi.ajp.159.9.1563

84. Salyers MP, Bonfils KA, Luther L, et al. The relationship between professional burnout and quality and safety in healthcare: a meta-analysis. J Gen Intern Med. 2017;32(4):475-482. doi:10.1007/ s11606-016-3886-9

85. Manh Than H, Minh Nong V, Trung Nguyen C, et al. Mental health and health-related quality-of-life outcomes among frontline health workers during the peak of COVID-19 outbreak in Vietnam: a cross-sectional study. Risk Manag Healthc Policy. 2020;13:2927-2936. doi:10.2147/RMHP.S280749

86. Wang W, Lu L, Kelifa MM, et al. Mental health problems in Chinese healthcare workers exposed to workplace violence during the COVID-19 outbreak: a cross-sectional study using propensity score matching analysis. Risk Manag Healthc Policy. 2020;13:2827-2833. doi:10.2147/RMHP.S279170

87. Kuoppala J, Lamminpää A, Liira J, Vainio H. Leadership, job well-being, and health effects - a systematic review and a meta-analysis. $J$ Occup Environ Med. 2008;50(8):904-915. doi:10.1097/JOM.0b013e31817e918d

88. Gewin V. Pandemic burnout is rampant in academia. Nature. 2021;591(7850):489-491. doi:10.1038/d41586-021-00663-2

89. Vinkers CH, Schaafsma FG. Burnout urgently needs robust research. Nature. 2021;592(7853):188. doi:10.1038/d41586-021-00896-1
Risk Management and Healthcare Policy

\section{Publish your work in this journal}

Risk Management and Healthcare Policy is an international, peerreviewed, open access journal focusing on all aspects of public health, policy, and preventative measures to promote good health and improve morbidity and mortality in the population. The journal welcomes submitted papers covering original research, basic science, clinical \& epidemiological studies, reviews and evaluations, guidelines, expert opinion and commentary, case reports and extended reports. The manuscript management system is completely online and includes a very quick and fair peer-review system, which is all easy to use. Visit http://www.dovepress.com/testimonials.php to read real quotes from published authors. 